\title{
Modified Bentall procedure with a rapid deployment valve in cardiac Behçet's disease: A case report
}

\author{
Chee-hoon Lee, MD, Hyung Gon Je, MD, PhD, and Min Ho Ju, MD
}

Video clip is available online.

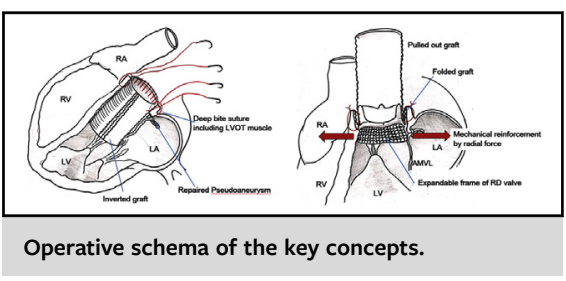

Cardiac involvement in Behçet's disease (BD) is rare; however, it can be a leading cause of death. ${ }^{1,2}$ Surgical treatments for cardiac BD are often challenging because of the pathophysiologic characteristics of vasculitis. ${ }^{1,3,4}$ The occurrence of postoperative anastomotic leakage and prosthesis detachment is well known, and in combination with repeated cardiac surgery, they often lead to a catastrophic situation. ${ }^{1,2,5}$

To reduce the risk of reoperation, some low-level evidence studies have proposed alternative surgical procedures, such as the Bentall procedure, instead of aortic valve replacement, ${ }^{1}$ or anchoring the aortic valve prosthesis into the left ventricular outflow tract (LVOT)., ${ }^{2,5}$

In this study, we report a novel surgical modification of Bentall operation for cardiac BD using the following 3 concepts: (1) complete resection of diseased aortic root tissue, (2) anchoring of the aortic graft at the LVOT muscle, and (3) further proximal reinforcement through the radial force of the rapid deployment $(\mathrm{RD})$ valve.

\section{CASE PRESENTATION}

A 50-year-old man with a 6-month history of progressive dyspnea and chest discomfort presented to the emergency department. Chest radiography showed pulmonary edema and marked cardiomegaly. He had a history of recurrent

\footnotetext{
From the Department of Cardiovascular and Thoracic Surgery, Research Institute for Convergence of Biomedical Science and Technology, Pusan National University Yangsan Hospital, Gyeongnam, Korea

Disclosures: The authors reported no conflicts of interest.

The Journal policy requires editors and reviewers to disclose conflicts of interest and to decline handling or reviewing manuscripts for which they may have a conflict of interest. The editors and reviewers of this article have no conflicts of interest.

Received for publication Feb 13, 2020; revisions received Feb 13, 2020; accepted for publication March 13, 2020; available ahead of print April 3, 2020.

Address for reprints: Min Ho Ju, MD, Department of Cardiovascular and Thoracic

Surgery, Pusan National University Yangsan, Hospital, Yangsan, Gyeongnam,

South Korea 626-770 (E-mail: deicidepan@naver.com).

JTCVS Techniques 2020;2:43-5

2666-2507

Copyright (C) 2020 The Authors. Published by Elsevier Inc. on behalf of The American Association for Thoracic Surgery. This is an open access article under the CC BY-NCND license (http://creativecommons.org/licenses/by-nc-nd/4.0/).

https://doi.org/10.1016/j.xjtc.2020.03.011
}

\begin{abstract}
CENTRAL MESSAGE
The Modified Bentall procedure using a subannular inverted graft and rapid deployment valve can be used to prevent prosthesis dehiscence in patients with car-
\end{abstract} diac BD. See Commentaries on pages 46 and 48.

oral aphthosis and scleritis. Echocardiography showed a left coronary cusp that was nearly detached from the aortic valve annulus, with mass-like conglomeration, and a pseudoaneurysm was observed at the ventriculo-aortic junction below the left coronary cusp (Figure 1, A). Moreover, marked aortic wall thickening with a saccular aneurysm from the left-right coronary commissure through the lesser curvature of the ascending aorta was also noted (Figure 1, $B$ ) on computed tomography. His preoperative C-reactive protein level and erythrocyte sedimentation rate were $2.94 \mathrm{mg} / \mathrm{dL}$ (normal range $<0.5 \mathrm{mg} / \mathrm{dL}$ ) and $57 \mathrm{~mm} / \mathrm{h}$ (normal range $<10 \mathrm{~mm} / \mathrm{h}$ ), respectively. Cardiac involvement in BD was strongly suspected. Ethical committee approval was waived because of the study nature. Informed consent for the publication was obtained from the patient.

\section{SURGICAL TECHNIQUE}

The patient underwent elective operation. Through a median sternotomy, a cardiopulmonary bypass procedure was performed, with transverse arch and bicaval cannulation. Under cardiac arrest, the ascending aorta and aortic roots were excised to the aortic valve annulus level as much as possible, and coronary buttons were created. The pseudoaneurysm at the ventriculo-aortic junction was repaired using 4-0 polypropylene double-layer continuous suture (Figure 2, A). To create a neosinus, a 30-mm Valsalva graft (Terumo Medical, Somerset, $\mathrm{NJ}$ ) cut to 8-cm length was inverted and placed in the LVOT, followed by deep 


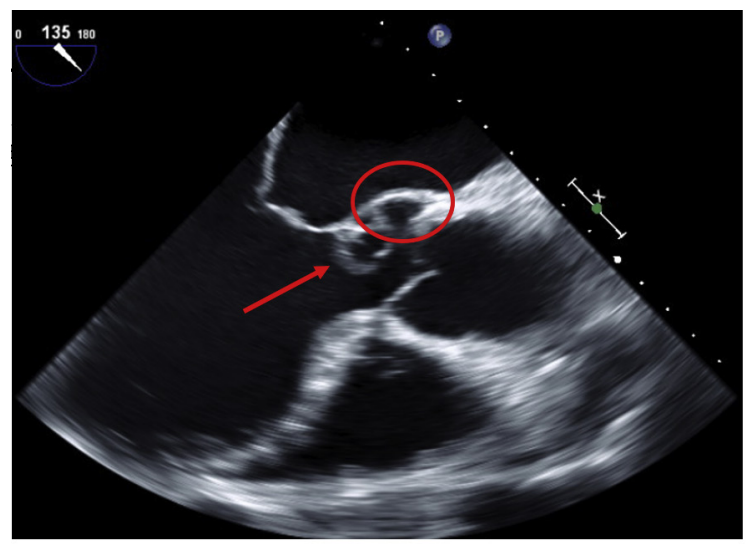

A

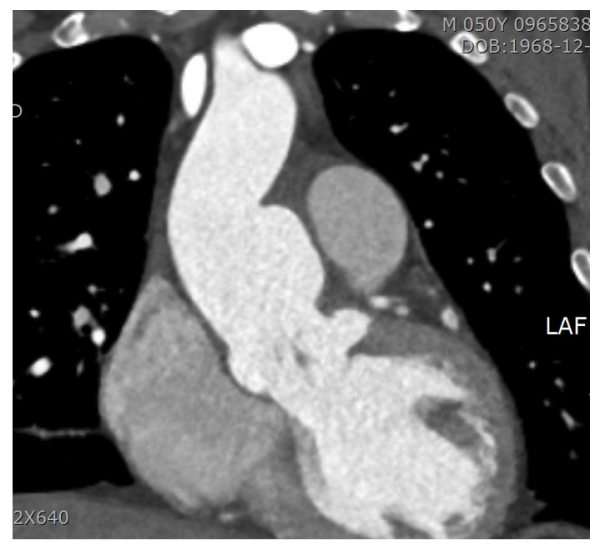

B

FIGURE 1. A, Preoperative midesophageal long-axis view of transesophageal echocardiography: The left coronary cusp was nearly detached from aortic valve annulus with a mass-like conglomeration (arrow), causing severe aortic valve regurgitation. A pseudoaneurysm was formed at the ventriculo-aortic junction below the left coronary cusp ( circle). All of these findings strongly suggested cardiac BD. B, Coronal section of preoperative computed tomography scan showed a saccular aneurysm from left-right coronary commissure through lesser curvature of ascending aorta with prominent inflammatory thickening of aortic wall by chronic vasculitis in cardiac BD.

bite polypropylene 3-0 double layer continuous suture including the LVOT muscle and 1-cm graft (Figure 2, B). To prevent narrowing of the neoannulus, a $25-\mathrm{mm}$ valve sizer was placed in the lumen when tying the anastomosis knot. After proximal anastomosis, the graft was pulled out, and 3 equidistant RD valve anchoring sutures were placed at the bottom of the folded graft. By using the suture as a guide, a 25-mm Intuity valve (Edwards Lifesciences LLC, Irvine, Calif) was inserted and carefully deployed such that the expandable frame was below the proximal end of the graft (Figure 2, C). Finally, coronary buttons were reimplanted, and distal anastomosis was performed under total circulatory arrest with moderate hypothermia (Video 1). The patient was transferred to the general ward 21 hours after surgery and was discharged on postoperative day 12 without complications. His echocardiography (Figure 3) and computed tomography findings were unremarkable until 9 months after surgery under immunosuppressive therapy, comprising methylprednisolone and methotrexate.

\section{DISCUSSION}

High surgical mortality associated with repeated aortic root surgery, because of prosthesis dehiscence or anastomosis, is the greatest concern for aortic valve procedures in cardiac BD. ${ }^{1,2}$ We attempted to resolve this problem on the basis of the advantages of other reported surgical techniques and using the concepts described.

The advantage of this procedure is the insertion of the inverted graft into the LVOT, which allows good visibility and
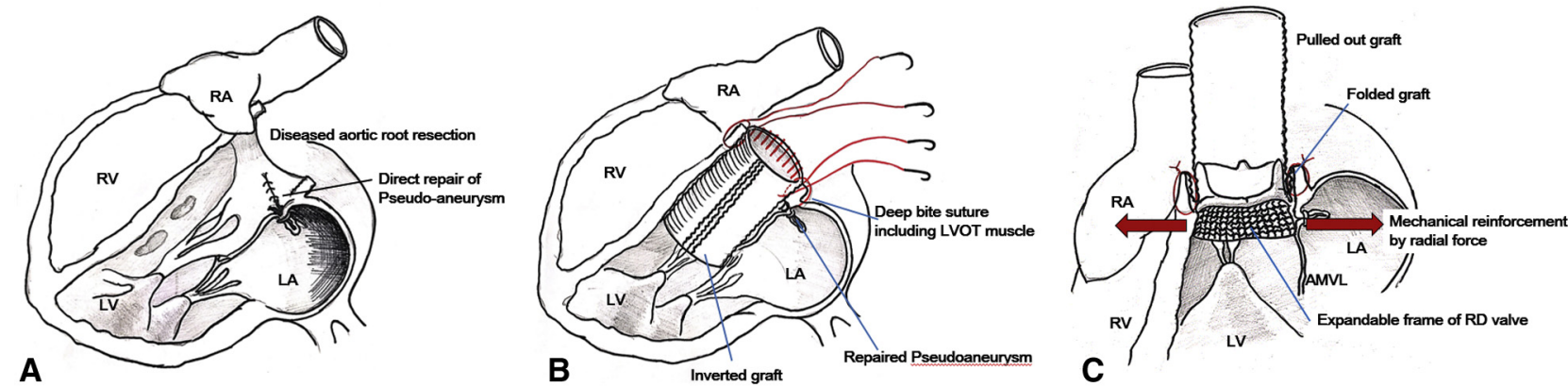

FIGURE 2. Operative schema. A, Ascending aorta, aortic root, and aortic valve were excised as much as possible after cardiac arrest by retrograde cardioplegia infusion. Thereafter, the pseudoaneurysm at the ventriculoaortic junction below the LCC was directly repaired using polypropylene continuous double-layer suture. B, A 30-mm Valsalva graft (Terumo Medical, Somerset, NJ) was cut to approximately $8 \mathrm{~cm}$ in length and inserted into the LVOT to ensure good visibility and easy configuration of anastomosis. The inverted aortic graft was fixed by continuous deep bite sutures including LVOT muscle and 1 -cm length of the aortic graft. C, After completion of the proximal anastomosis, the inverted aortic graft was pulled out. Then the 25-mm RD valve (Intuity, Edwards Lifesciences LLC, Irvine, Calif) was implanted at the bottom of the graft so that the expandable frame of the RD valve was positioned lower than the proximal end of the graft. In this way, the proximal anastomosis line was further reinforced by the radial force of the expandable frame of the RD valve. $R A$, Right atrium; $R V$, right ventricle; $L V$, left ventricle; $L A$, left atrium; $L V O T$, left ventricular outflow tract; $A M V L$, anterior mitral valve leaflet; $R D$, rapid deployment. 


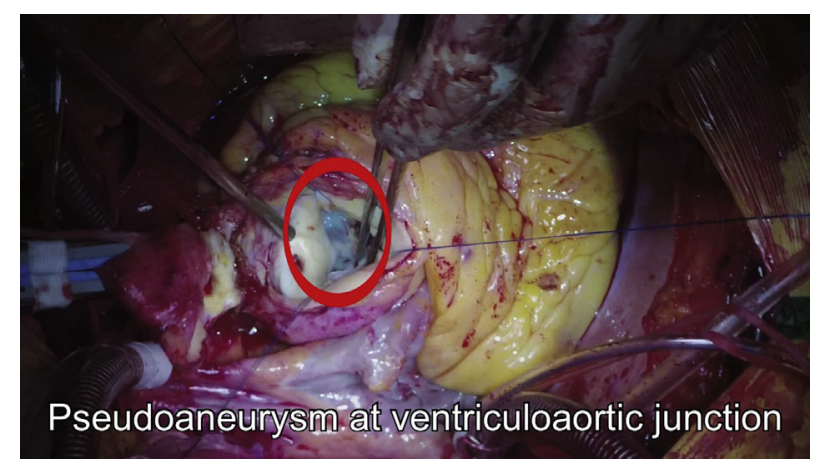

VIDEO 1. After inducing cardiac arrest using retrograde cardioplegia infusion, the ascending aorta and the aortic root were excised as much as possible, the aortic valves were resected, and coronary buttons were made. Subsequently, the pseudoaneurysm at the ventriculoaortic junction is directly repaired with 4-0 polypropylene continuous double layer suture. Then, the 30-mm Valsalva graft (Terumo Medical, Somerset, NJ) was cut to approximately $8 \mathrm{~cm}$ in length, inverted and placed in the LVOT. and the configuration for anastomosis was adjusted. Thereafter, the proximal anastomosis was made between the graft and LVOT muscle with deep bite suture using double-layered 3-0 polypropylene continuous suture. To prevent narrowing of the neo-annulus, a 25-mm valve sizer was placed in the lumen when tying the anastomosis knot. After proximal anastomosis was completed, the graft was pulled out again and a valve anchoring suture was performed equidistance to the bottom of the folded graft. By using the suture as a guide, a 25-mm Intuity valve (Edwards Lifesciences LLC, Irvine, Calif) was inserted and carefully deployed such that the expandable frame was below the proximal end of the graft. After reimplantation of the coronary buttons, the aortic clamp was released under total circulatory arrest. Distal anastomosis was performed after complete resection of aorta with inflammatory changes. LVOT, Left ventricular outflow tract. Video available at: https://www.jtcvs.org/article/S2666-2507(20)30159-0/ fulltext.

proper configuration before anastomosis. Therefore, the hemostatic line between LVOT and the aortic graft is easily secured. Another advantage is that the radial force of the expandable frame of the RD valve can be used to further reinforce the proximal suture line, decreasing prosthesis dehiscence.
There are some disadvantages to this procedure. Deep bite stitches on the aortic subannular structure may present a risk of rhythm disturbance; however, it did not occur in our patient. There is a probability of requirement for repeated valve surgery on implanting the tissue valve at a relatively young age. Thus, a large-sized $(25 \mathrm{~mm})$ valve prosthesis was implanted to enable future valve-in-valve transcatheter aortic valve replacement. Although this technique seems suitable for BD and other inflammatory aortic root pathologies, it may be inappropriate in other circumstances such as aortic root disruption by infective endocarditis.

\section{CONCLUSIONS}

The modified Bentall procedure using a subannular inverted graft and rapid deployment valve can be applied to prevent prosthesis dehiscence in patients with cardiac BD. Long-term follow-up is required.

All individuals with involvement in the study were included in authorship. The authors thank Editage (www.editage.co.kr) for English language editing.

\section{References}

1. Ghang B, Kim JB, Jung SH, Chung CH, Lee JW, Song JM, et al. Surgical outcomes in Behcet's disease patients with severe aortic regurgitation. Ann Thorac Surg. 2019;107:1188-94.

2. Jung Y, Ahn BH, Lee KS, Jeong IS, Kim KH, Na KJ, et al. A novel solution to prosthetic valve dehiscence after aortic valve surgery in Behcet's disease. Interact Cardiovasc Thorac Surg. 2017;24:342-7.

3. Koo HJ, Yang DH, Kang JW, Han K, Chung CH, Song JK, et al. Demonstration of prosthetic aortic valve dehiscence in a patient with noninfectious aortitis by multimodality imaging: findings of echocardiography and computed tomography. Circulation. 2013;128:759-61.

4. Song JK, Kim MJ, Kim DH, Song JM, Kang DH, Lee I, et al. Factors determining outcomes of aortic valve surgery in patients with aortic regurgitation due to Behcet's disease: impact of preoperative echocardiographic features. J Am Soc Echocardiogr. 2011;24:995-1003

5. Azuma T, Yamazaki K, Saito S, Kurosawa H. Aortic valve replacement in Behcet's disease: surgical modification to prevent valve detachment. Eur J Cardiothorac Surg. 2009;36:771-2.

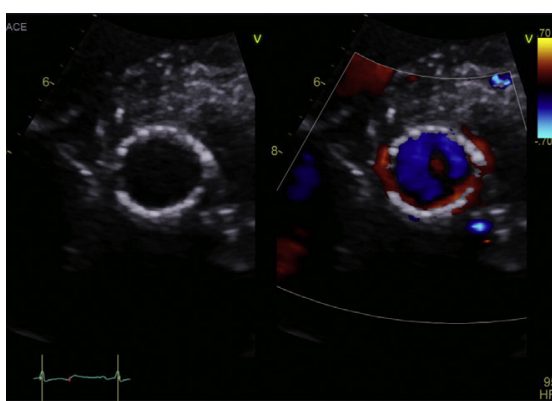

A

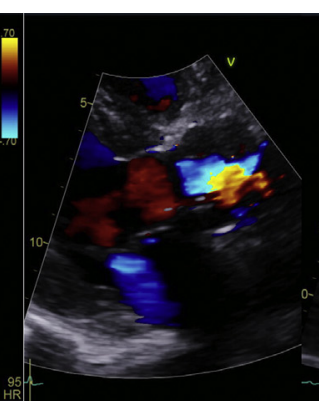

B

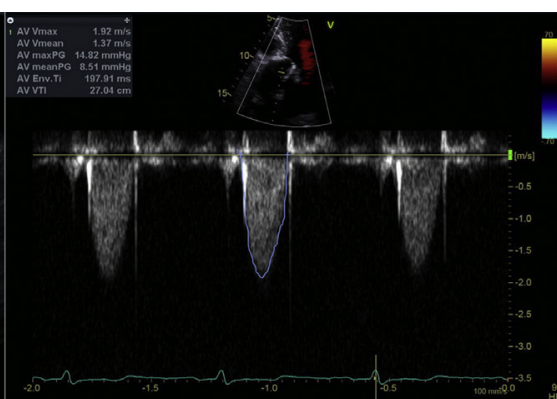

C

FIGURE 3. Postoperative findings of transthoracic echocardiography: A, Parasternal short-axis view showed unremarkable prosthetic aortic valve position without paravalvular leakage. B, Parasternal long-axis view showed that the anterior mitral valve leaflet motion was not interfered by the expandable frame of rapid deployment valve despite of subannular valve positioning. C, Continuous-wave Doppler under apical 5-chamber view revealed that peak transaortic pressure gradient and mean transaortic pressure gradient were $14.8 \mathrm{~mm} \mathrm{Hg}$ and $8.5 \mathrm{~mm} \mathrm{Hg}$, respectively. 